\title{
Praxis und Erkenntnis
}

\author{
Harald Walach ${ }^{a}$ Andreas Michalsen ${ }^{b}$ \\ a School of Social Sciences \& Samueli Institute for Information Biology, University of Northampton, UK \\ ${ }^{b}$ Innere Medizin V und Integrative Medizin, Kliniken Essen-Mitte, Deutschland
}

Mit diesem Heft halten Sie die erste Ausgabe der neuen «Forschenden Komplementärmedizin» in der Hand. Gleichzeitig mit der Neugestaltung des Umschlags haben wir uns entschlossen, künftig im Titel auf «Klassische Naturheilkunde» zu verzichten. Das inhaltliche Spektrum der Zeitschrift umreisst der neue Untertitel «Wissenschaft - Praxis - Perspektiven». Besonders wichtig ist uns ein verstärkter Praxisbezug. Man hört immer wieder aus Kreisen der Komplementärmedizin, man könne an einzelnen Patienten Erkenntnis darüber gewinnen, ob eine Intervention ursächlich für eine Verbesserung war [1]. Die orthodoxe Methodenlehre widerspricht dem vehement, unter anderem weil die Zeit als potentiell verändernder Faktor und die Intervention selbst unauflöslich miteinander verquickt sind. Es ist nicht von der Hand zu weisen, dass grosse wissenschaftliche Fortschritte oft aus sorgfältiger Beobachtung einzelner Fälle erwachsen sind. Denken wir nur an Jenners Entdeckung des Zusammenhangs zwischen Kuhpocken und Pockenimmunität bei Melkerinnen.

Der erste wichtige Schritt wissenschaftlicher Theorienbildung beginnt immer mit der Betrachtung verschiedener einzelner Ereignisse, die ein grundlegendes Muster nahe legen, und mit der intuitiven Verknüpfung dieser einzelnen Erfahrung zu einem vermutlichen kausalen Muster. Weitere Schritte müssen aber folgen, wenn diese erste, hypothetische Struktur auf Gültigkeit geprüft werden soll [2]. Meistens führen weitere Prüfungen $\mathrm{zu}$ einer Revision der ursprünglich vermuteten Struktur von Zusammenhängen, etwa der von Ursache und Wirkung. Die konventionelle Methodenlehre befasst sich meistens und fast ausschliesslich damit, wie bereits vorhandene anfängliche Hypothesen und Theorien auf Gültigkeit geprüft werden können und zwar so, dass die gewonnene Erkenntnis nicht nur einigermassen stimmt, sondern auch breit anwendbar ist. Sie hat also einen robusten, grossen Wirkungsbereich vor Augen. Die Erkenntnis hingegen, die sich aus Einzelfällen ableitet, ist diesem Verallgemeinerungsschritt der
Methodenlehre entweder vorgelagert oder aber nachfolgend, indem die einmal verbreitete Erkenntnis auf ihre Brauchbarkeit in der Praxis geprüft wird.

In jedem Fall beginnt und endet die wissenschaftliche Bemühung um Erkenntnis in der Praxis bzw. am Einzelfall. Der Praktiker also ist nicht nur als Konsument von Erkenntnis bedeutsam, wie dies die Bewegung der evidenzbasierten Medizin nahe legt, sondern auch als Quelle von Erkenntnis. Heuristisch besonders wertvoll sind dabei immer diejenigen Fälle, die eben genau nicht mit dem erwarteten Verlauf übereinstimmen. Werden unsere Erwartungen nicht erfüllt, dann wissen wir, dass unser Modell von der Wirklichkeit nicht stimmt. Einzelfälle liefern uns aber auch das Rohmaterial für neue Hypothesen und Modelle. Konkrete Fallbeispiele sind also, erkenntnistheoretisch gesehen und bildlich gesprochen, das Fleisch, das unser Knochengerüst der Hypothesen und Modelle zu einem griffigen, sinnenfälligen Körper macht und uns zeigt, wo noch Strukturen fehlen.

Wir wollen diese bedeutende Rolle der Praxis künftig verstärkt würdigen, indem wir regelmässig ausführliche Falldarstellungen veröffentlichen: Klassischerweise wurden Fallberichte genutzt, um neuartige diagnostische oder therapeutische Befunde mitzuteilen. Weitere Inhalte bildeten Fallberichte zur Ausbildung und Erläuterung. Schliesslich sind Fallberichte zwingend anerkannt zur Mitteilung von unerwünschten Wirkungen und sind damit die Basis für die systematische Registrierung von Arzneimittelnebenwirkungen. Dennoch haben in den letzten Jahren nur noch wenige Journals Fallberichte als Publikationsform weitergeführt. Inzwischen wurden Fallberichte unter der Rubrik «Evidence based case reports» vom British Medical Journal (BMJ) wieder eingeführt. Diese Rubrik soll der Problematik Rechnung tragen, dass die an Populationen gewonnenen Daten beim einzelnen Patienten häufig gar nicht so einfach angewendet werden können. Fallberichte des BMJ beschreiben somit den Fall im

\begin{tabular}{ll}
\hline KARGER & ( ) 2006 S. Karger GmbH, Freiburg \\
Fax +497614520714 & Accessible online at: \\
$\begin{array}{l}\text { E-mail Information@Karger.de } \\
\text { www.karger.com }\end{array}$ & www.karger.com/fok
\end{tabular}

Prof. Dr. Dr. Harald Walach

University College Northampton

School of Social Sciences \& Samueli Institute for Information Biology

European Office Boughton Green Rd, UK-Northampton NN2 7AL

Tel. +44 1604-892952, Fax -722067

E-mail harald.walach@northampton.ac.uk 
Spannungsfeld der dazu vorliegenden wissenschaftlichen Daten. In der Komplementärmedizin sind Fallberichte unseres Erachtens noch wichtiger. Patienten in naturheilkundlicher Behandlung sind oft durch ein spezifisches Behandlungsinteresse und besondere persönliche Erfahrungen geprägt. Die Behandler haben vielfältige Therapiemethoden zur Verfügung und unterschiedliche Sichtweisen von Möglichkeiten und Grenzen der Behandlung und der Indikation im Gesamtbehandlungskonzept. Wir stellen uns daher vor, dass die Fallberichte in der «Forschenden Komplementärmedizin» auf folgende Aspekte ein besonderes Augenmerk richten:

- Besondere Wirksamkeit, Indikation oder unerwünschte Wirkungen.

- Besonderheit der therapeutischen Situation in der Naturheilkunde und Komplementärmedizin.

- Weiterbildende, praktische Anwendung eines Verfahrens.

Beim Abfassen der Fallberichte sollten folgende Punkte beachtet werden:

1. Das Patienteneinverständnis zur Veröffentlichung der Daten muss vorliegen, und der Fall muss so anonymisiert werden, dass die Person weder durch (Vor-)Namen, Beruf, Geburtsdatum noch durch andere Besonderheiten erkannt werden kann.

2. Der diagnostische Befund sollte präzise dargestellt werden (sowohl konventionell als auch naturheilkundlich).

3. In der Diskussion sollte eine kritische Stellungnahme erfolgen.

4. Eine Schlussfolgerung sollte die Hauptaussage des Fallberichts zusammenfassen.

5. Die Darstellung soll vier Druckseiten einschließlich deutscher Zusammenfassung und englischem Summary nicht überschreiten.
Mit der neuen Praxisrubrik greifen wir einen oft geäusserten Wunsch auf und hoffen sehr, dass Sie, liebe Leserinnen und Leser, diese Gelegenheit auch beim Schopf packen. Was für die Wissenschaft gilt, gilt auch für die Praxis: Ein nicht publiziertes Forschungsergebnis ist verloren. Ein nicht publizierter, aussergewöhnlicher Fall wurde nie behandelt. Erst wenn wir uns die Mühe machen, unsere Gedanken zu fassen, gewinnen sie Gestalt, Dauer und Wirkung. In diesem Sinne appellieren wir also an alle Praktiker - aus allen Schulrichtungen, aus allen Ländern, aus allen Disziplinen - ihre Fälle einzureichen. Auch eingereichte Fallberichte gehen durch ein (vereinfachtes) Review-Verfahren. Dabei prüft die Schriftleitung, ob wichtige Informationen fehlen und nicht zuletzt, ob der Fall auch anregend geschrieben ist. Denn wir wollen nicht einfach erbauend oder belehrend sein, sondern auch im besten Sinne unterhalten.

Ein erstes Fallbeispiel, das aus unserer Sicht die Kunst der Kasuistik gut verkörpert, finden Sie in diesem Heft [3]. Der Fall ist plastisch geschildert und enthält alle notwendigen Informationen. Er kaschiert auch nicht die Ambivalenz, die ihn prägte und die kennzeichnend ist für die Praxis. - Wir wünschen Ihnen eine anregende und bereichernde Lektüre.

\section{Literatur}

1 Kienle GS, Karutz M, Matthes H, Matthiesen P, Petersen P, Kiene H: Evidenzbasierte Medizin: Konkurs der ärztlichen Urteilskraft? Dtsch Ärztebl 2003;100: A 2142-2146.

2 Walach H: Psychologie: Wissenschaftstheorie, philosophische Grundlagen und Geschichte. Stuttgart, Kohlhammer, 2005.

3 Teut M, Warning A: Knochenmetastasen bei Mamma-Karzinom. Forsch Komplementärmed DOI: 10.1158/000091095. 